\title{
Spontaneous pneumothorax due to metastatic carcinoma of the rectum
}

\author{
P E BEARN, O J LAU \\ From the Regional Cardiothoracic Unit, Brook General Hospital, London
}

Spontaneous pneumothorax is a rare complication of both primary and secondary malignant tumours of the lung. We report the first case caused by metastatic carcinoma of the rectum.

\section{Case report}

A 59 year old man presented with left sided chest pain of sudden onset. He gave a six month history of dry cough with one episode of haemoptysis. Ten years previously he had undergone an abdominoperineal resection for a Duke's C adenocarcinoma of the rectum. A spontaneous pneumothorax was diagnosed and confirmed by a chest radiograph which also suggested a left hilar abnormality (figure).

The pneumothorax was managed by intercostal chest drainage but the lung failed to re-expand. Bronchoscopy showed a tumour in the left main bronchus close to its bifurcation. Biopsy of this lesion revealed a poorly differentiated adenocarcinoma. Other investigations showed no contraindication to resection.

At operation the left lung was found to be totally collapsed with a $4 \times 5 \mathrm{~cm}$ tumour in the lower lobe, infiltrating close to the visceral pleura at the base. As the tumour extended

Address for reprint requests: Mr O J Lau, FRCs, Regional Cardiothoracic Unit, Brook General Hospital, London SE18 4LW.

Accepted 10 November 1987

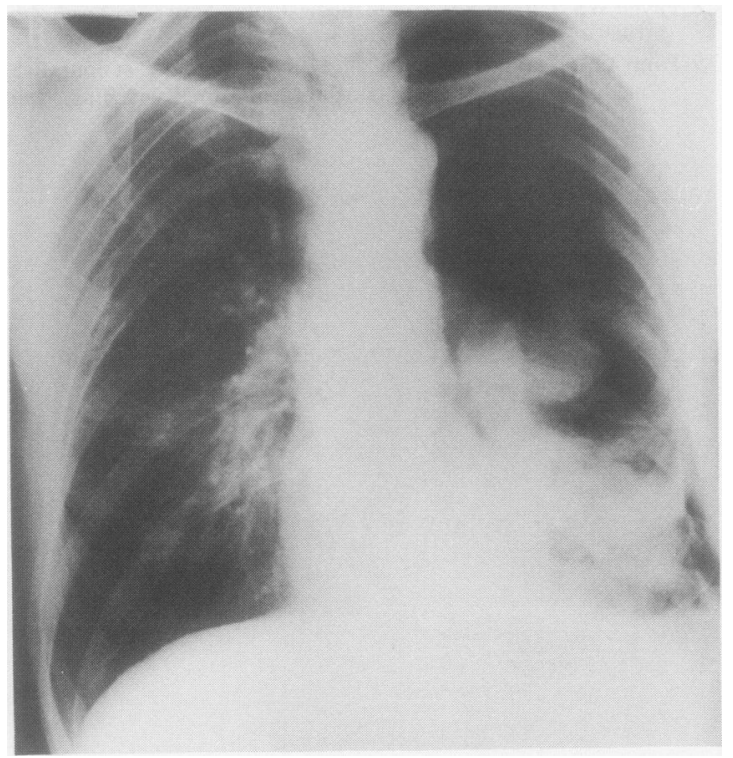

Penetrated posteroanterior chest radiograph showing a left pneumothorax. proximally to affect the upper lobe, a left pneumonectom was performed. Microscopic examination of the specime showed a poorly differentiated adenocarcinoma, consisten $\vec{k}$ with a metastasis from the rectal carcinoma.

There were no postoperative complications and the patiento was alive and well two years after resection.

\section{Discussion}

Spontaneous pneumothorax associated with secondary neoplasia in the lung is uncommon and carries a poor prognosis In the present case the pneumothorax was probably caused by breakdown of the tumour directly into the pleural space $\mathbb{D}$ Where possible surgery, which offers the only hope of long term survival, should be undertaken.

Spontaneous pneumothorax may be caused by primary of secondary neoplasia in the lung. Of all metastatic tumours osteogenic sarcoma has been reported to cause pneumo thorax most frequently. Others include Wilm's tumour Ewing's tumour, ${ }^{1}$ melanoma, ${ }^{2}$ and endometrial adeno carcinoma. $^{3}$

Many of these patients belong to an age group in which underlying lung disease, such as chronic bronchitis and emphysema, are common. A tumour may partially obstruct the lumen of a bronchus, exerting a flap valve effect, to cause further emphysematous lung changes and bullae formation. distal to the obstruction. Compensatory emphysema and bullous changes may also occur adjacent to a collapsed segment of the lung, a result of complete occlusion of bronchial lumen by the tumour. Rupture of a bulla, spontan' eously or by sudden increase of intrabronchial pressure as ip. coughing or sneezing, will produce a spontaneous pneumo thorax. Rarely, a peripheral necrotic tumour may rupture directly into the pleural space, creating a bronchopleura fistula and spontaneous pneumothorax.

The prognosis of these patients has been poor. Generall they are managed by intercostal drainage, and only a few. with primary neoplasia are suitable for resection. For thosed treated conservatively life expectancy has ranged from a few days to a few months. ${ }^{4}$ The present case appears to be the first reported case of lung metastasis from a rectal tumout presenting as a spontaneous pneumothorax, with successfutb treatment by resection.

\section{References}

1 D'Angio GJ, Iannaccone G. Spontaneous pneumothorax as complication of pulmonary metastases in malignant tumours of childhood. American Journal of Roentgenology, Radiun Therapy and Nuclear Medicine 1961;86:1092-102.

2 Yeung KY, Bonnet JD. Spontaneous pneumothorax with metå static malignant melanoma. Chest 1977;71:435-6.

3 Helmkamp BF, Beecham JB, Wandtke JC, Keys H. Spontaneous pneumothorax in gynecological malignancies. Am J Obstex Gynecol 1982;142:706-7.

4 Heimlick HJ, Rubin M. Spontaneous pneumothorax as a present ing feature of primary carcinoma of the lung. Dis Che 1955;27:457-64. 